\title{
16-098 - Nuclear Nonproliferation Applications of 14C Analyses by Accelerator Mass Spectrometry
}

Mathew Snow, John Olson, Mary Adamic, Matthew Watrous, John Southon

November 2016

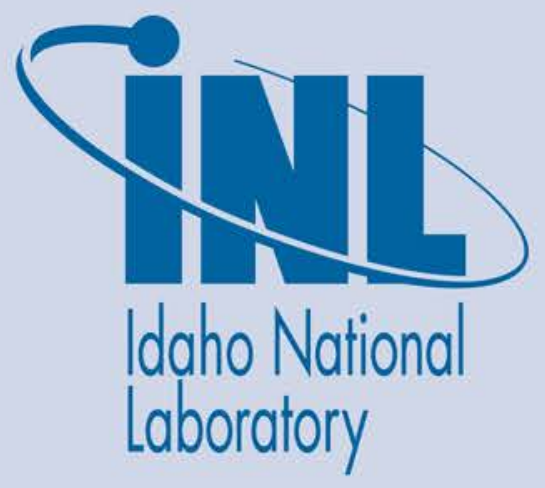

The INL is a U.S. Department of Energy National Laboratory operated by Battelle Energy Alliance 


\title{
16-098 - Nuclear Nonproliferation Applications of 14C Analyses by Accelerator Mass Spectrometry
}

\author{
Mathew Snow, John Olson, Mary Adamic, Matthew Watrous, John Southon
}

November 2016

\begin{abstract}
Idaho National Laboratory
Idaho Falls, Idaho 83415
\end{abstract}

http://www.inl.gov

Prepared for the

U.S. Department of Energy

Assistant Secretary for __, OR Office of

Under DOE Idaho Operations Office

Contract DE-AC07-05ID14517 


\title{
16-098 - Nuclear Nonproliferation Applications of ${ }^{14} \mathrm{C}$ Analyses by Accelerator Mass Spectrometry
}

\author{
Mathew Snow $^{1}$, John Olson ${ }^{1}$, Mary Adamic ${ }^{1}$, Matthew Watrous ${ }^{1}$, John Southon ${ }^{2}$ \\ 1-Idaho National Laboratory \\ 2-University of California-Irvine
}

\section{General Project Description:}

In January 2015 the Idaho National Laboratory installed a 0.5 MV Accelerator Mass Spectrometer (AMS). The INL AMS is ideally suited for ultra-trace isotopic analyses supporting national security applications including nuclear forensics and nonproliferation. This LDRD seeks to develop INL's sample preparation and AMS analysis capability to analyze the isotopic composition of carbon containing samples for ultratrace ${ }^{14} \mathrm{C}$.

\section{Summary:}

FY-16 efforts were dedicated to 2 general categories: 1) INL staff training at UC-Irvine and construction of an INL ${ }^{14} \mathrm{C}$ sample preparation manifold system, 2) Preliminary method development and validations of INL's sample preparation capabilities for simple organic samples.

\section{1) INL Staff Training and ${ }^{14} \mathrm{C}$ Sample Preparation Manifold Construction:}

In FY-16 INL scientists received training in current state-of-the-art ${ }^{14} \mathrm{C}$ AMS sample preparation techniques under the direction of UC-Irvine scientists Dr. John Southon and Dr. Xaimei Xu (leading experts in ${ }^{14} \mathrm{C}$ AMS analyses). Following this training, the INL team developed and constructed a gas phase ${ }^{14} \mathrm{C}$ sample preparation manifold system (Figure 1). Construction of this system was required in order to stand up the ultra-trace ${ }^{14} \mathrm{C}$ AMS analysis capability at INL. The current manifold configuration has a combustion line for conversion of organic samples to $\mathrm{CO}_{2}$ and a graphitization line for cryogenic purification of $\mathrm{CO}_{2}$ prior to sample graphitization and ${ }^{14} \mathrm{C}$ AMS analysis.

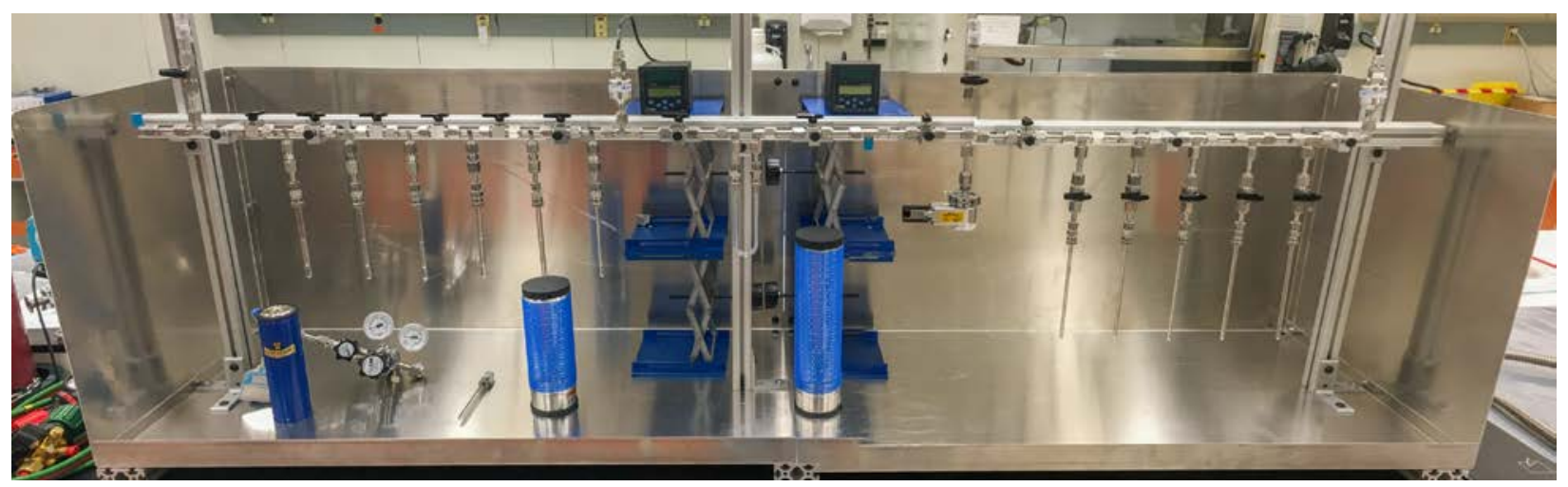

Figure 1. INL's ${ }^{14} \mathrm{C}$ sample preparation manifold system constructed under this LDRD. 

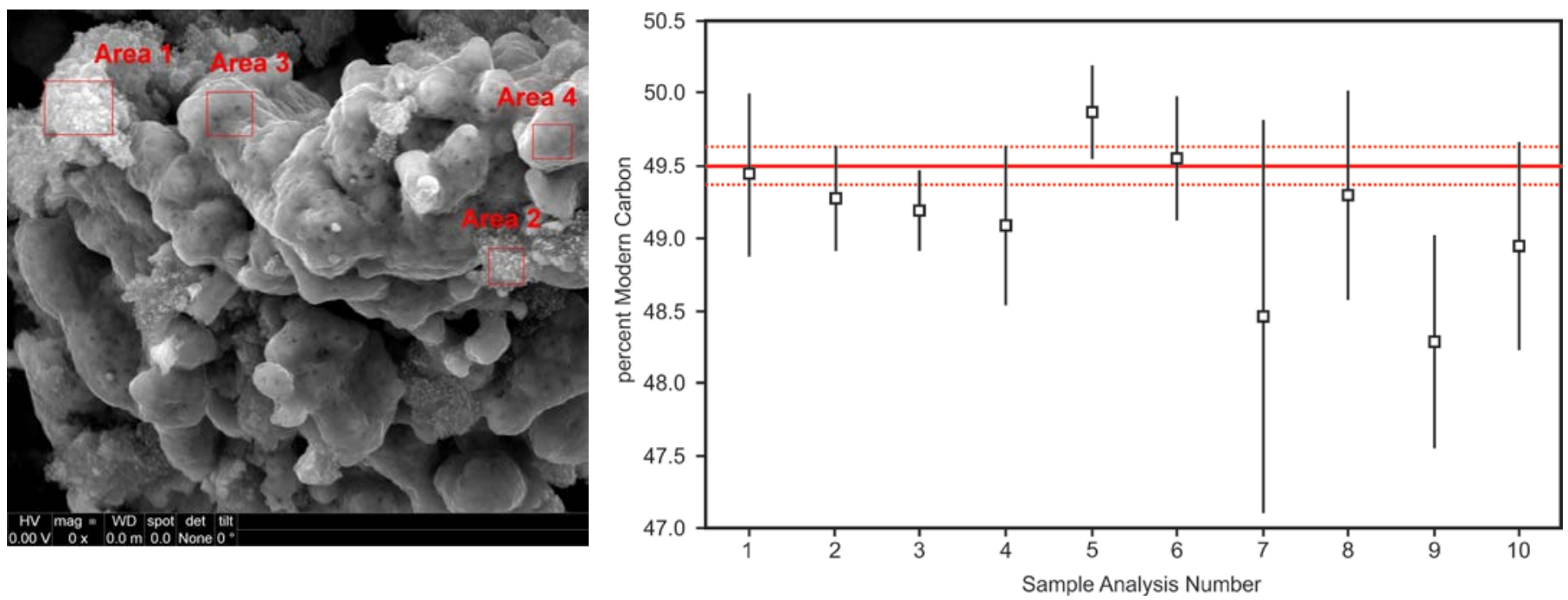

Figure 2. (left) SEM micrographs showing the formation of purified carbon species on the surface of an iron catalyst. (right) Preliminary ${ }^{14} \mathrm{C}$ analysis data for the standard IAEA-C7 (oxalic acid). Red solid and dashed lines represent the certificate value and associated uncertainty. INL measurements show production of high yields of purified carbon with the correct ${ }^{14} \mathrm{C}$ content.

\section{2) Preliminary Evaluation of Analysis Capabilities for Organic Samples}

Preliminary method development and validations for analyses of simple organic samples were performed in FY-16 using IAEA and NIST oxalic acid standards, with the current optimized method results shown in Figure 2. INL measurements for IAEA-C7 are in agreement with the certificate value for this material, demonstrating initial success in standing up the ultra-trace ${ }^{14} \mathrm{C}$ AMS sample preparation and analysis capability at INL. Further refinements in the measurement precision as well as extension of current analysis capabilities to include more complex matrices (including complex environmental samples, inorganic samples, and air samples) will continue throughout the lifecycle of this project.

\section{Benefits to DOE:}

${ }^{14} \mathrm{C}$ sample preparation/analysis capability development at INL enables application of this isotope to support a variety of National and Homeland Security missions including nuclear forensics and nonproliferation. Additional potential future applications of this capability range from long term environmental stewardship supporting advanced nuclear fuel cycles, clean alternative energy sources, and evaluations of global climate change.

\section{Presentations:}

Snow, M.S. Isotopic Signature and Standards Development Supporting Nuclear Forensics and Nonproliferation. National Technical Nuclear Forensics Academic/National Laboratory Collaboration Meeting. Los Alamos, NM. August 3 ${ }^{\text {rd }}, 2016$. 